$\mathrm{G}$ ass transi ti on of crossl i nked pol ystyrene shel Is for ned on the surface of cal ci um car bonate whi sker

\begin{tabular}{|l|l|}
\hline 著者 & $\begin{array}{l}\text { SASAK Takashi, KAWAGOE Shoko, M TSUYA Haj i me, } \\
\text { I RI E Sat oshi, SAKURAl Kensuke }\end{array}$ \\
\hline $\begin{array}{l}\text { j our nal or } \\
\text { publ i cat i on ti tl e }\end{array}$ & $\begin{array}{l}\text { Journal of pol yner sci ence. Part B: Pol ymer } \\
\text { physi cs }\end{array}$ \\
\hline vol une & 44 \\
\hline nunber & 17 \\
\hline page r ange & $2475-2485$ \\
\hline year & $2006-09$ \\
\hline URL & ht t p: //hdl . handl e. net /10098/1767 \\
\hline
\end{tabular}




\title{
Glass Transition of Crosslinked Polystyrene Shells Formed on the Surface of Calcium Carbonate Whisker
}

\section{TAKASHI SASAKI, SHOKO KAWAGOE, HAJIME MITSUYA, SATOSHI IRIE, KENSUKE SAKURAI}

Department of Materials Science \& Engineering, University of Fukui, Fukui 910 8507, Japan

Correspondence to: T. Sasaki (E-mail: sasaki@matse.fukui-u.ac.jp).

\begin{abstract}
Glass transition of core/shell capsules consisting of calcium carbonate whisker as a core and crosslinked polystyrene as a shell was studied by differential scanning calorimetry (DSC). The thickness of the crosslinked shell was in the range of $26-81 \mathrm{~nm}$. The crosslinked shells were revealed to show higher glass transition temperatures $\left(T_{\mathrm{g}}\right)$ than the corresponding bulk values. It was revealed that a thicker shell exhibits a lower $T_{\mathrm{g}}$ than a thinner shell, and that capsules without core (hollow capsules) exhibit lower $T_{\mathrm{g}}$ 's than the corresponding core/shell capsules. These results suggest that the interfacial molecular interaction plays a role in the segmental relaxation which is responsible for the glass transition. The difference in $T_{\mathrm{g}}$ between the core/shell and hollow samples was reduced when a coupling agent, methacrylic acid 3-(tri-methoxysilyl)propyl ester was not included. This also suggests the interfacial effect on $T_{\mathrm{g}}$. However, the results still suggest that the enhancement of $T_{\mathrm{g}}$ for the present crosslinked shells is not only due to the interfacial effect but also to the effects of chain configuration and heterogeneous crosslink.
\end{abstract}

Keywords: calcium carbonate whisker; core/shell capsules; crosslinked polystyrene; DSC; glass transition

\section{INTRODUCTION}

Since the different glass transition behavior for polymer ultrathin films from that of the bulk material was reported, the dynamics in nano-sized polymeric materials has been attracted particular attention, and many attempts to understand the nature of this intriguing phenomenon have been done. ${ }^{1-7}$ The subject is important not only for the physicists studying the nature of glass-forming liquids, but also for developments of nano-sized materials for practical use in various fields. In particular, nano-sized core/shell capsules have been extensively studied ${ }^{8-10}$ because of their applicability to drug delivery system. ${ }^{11}$ However, the physical properties, e.g., glass transition, melting, and structural relaxation of nano-sized materials other than thin films have not 
yet been well-investigated.

Recently, we prepared polymeric rod-like core/shell and hollow capsules using calcium carbonate whisker as a core substance. ${ }^{12}$ Polymeric shells of which the thicknesses were less than $100 \mathrm{~nm}$ were formed on the surface of rod-like calcium carbonate particles (whisker) via emulsion polymerization. The glass transition temperatures $\left(T_{\mathrm{g}}\right)$ of such thin polymer shells were revealed to be almost identical to the bulk values, and no size effect on $T_{\mathrm{g}}$ was observed. ${ }^{12} \quad T_{\mathrm{g}}$ values identical to the bulk value was also reported for polystyrene microspheres of which the diameters were several tens of nanometers. ${ }^{13-15}$ In contrast, ultrathin films exhibit $T_{\mathrm{g}}$ 's which depend significantly on the thickness in the range below $100 \mathrm{~nm}$.,4,7,16,17 The origin of the above different glass transition behavior for the capsules and particles from ultrathin films might be attributed to the difference in the chain configuration in different geometries, but the molecular mechanism is not clear at the present time.

As for supported ultrathin films, $T_{\mathrm{g}}$ also depends on the chemistry of substrate, suggesting that the molecular interaction at the interface plays an important role in determining segmental mobility. ${ }^{16,18,19}$ In this study, we have found that core/shell capsules of which the shells consist of crosslinked polystyrene (shell crosslinked capsules) show higher $T_{\mathrm{g}}$ 's than the corresponding hollow capsules. This suggests that the interaction at the core/shell interface affects $T_{\mathrm{g}}$ for the crosslinked shell. In contrast, non-crosslinked shells exhibited $T_{\mathrm{g}}$ 's almost identical to those of the hollow capsules as we reported previously. ${ }^{12}$ It is likely that crosslink affects $T_{\mathrm{g}}$ in a particular manner in ultrathin layer, though the molecular mechanism is not known at present: it might enhance the compatibility (molecular interaction) between the core and shell at the interface, and/or there might be some configuration effects which are specific to the ultrathin layer of crosslinked polymer.

Here, we report a calorimetric study on the glass transition behaviors of the above shell crosslinked capsules with a rod-like shape. Differential scanning calorimetry (DSC) of the step-scan mode was used to evaluate $T_{\mathrm{g}}$ 's of the polymeric capsules. We prepared rod-like core/shell and hollow capsules which consisted of a crosslinked polystyrene shell with different degrees of crosslink and a calcium carbonate core. Effects of size and core/shell interface were also studied on samples with different shell thicknesses and with different status of the interface by using a coupling agent.

\section{EXPERIMENTAL}

\section{Preparation of Shell Crosslinked Capsules}

The capsules were prepared via emulsion copolymerization of styrene and 
divinylbenzene (DVB) in a suspension of calcium carbonate whisker particles. ${ }^{12}$ Styrene and DVB were distilled under reduced pressure before use. Calcium carbonate whisker (BS-P) was supplied from Maruo Calcium Co. The diameter of the whisker ranged from 300 to $600 \mathrm{~nm}$, and the length ranged from 1.0 to $3.0 \mu \mathrm{m}$. To improve the compatibility between the core/shell interface, we added a small amount of coupling agent, methacrylic acid 3-(tri-methoxysilyl)propyl ester (MAMSP). ${ }^{20}$ We prepared samples with different degrees of crosslink as shown in Table 1. We also prepared a sample with a thicker shell by introducing a larger amount of monomers with respect to BS-P (PSW5S), and to investigate the influence of the interfacial status on $T_{\mathrm{g}}$ a sample without the coupling agent MAMSP was prepared (PSW5N). Emulsion polymerizations were performed by using sodium peroxodisufate as an initiator. Detailed procedure was the same as that described previously. ${ }^{12}$ Typical emulsion mixture at the final stage of reaction consisted of $2.0 \mathrm{~g}$ of monomers, $50 \mathrm{mg}$ of laurylbenzenesulfonic acid sodium salt, $4.0 \mathrm{~g}$ of BS-P, and $68 \mathrm{~g}$ of distilled water. After the completion of polymerization, insoluble materials were separated by filtration, and washed with methanol for more than 20 times: this was needed to remove microspheres which were formed simultaneously during the reaction. The removal of spheres was checked for all the samples by transmission electron microscopy (TEM). Finally, the yielded capsules were dried under vacuum at room temperature for more than $72 \mathrm{~h}$.

From the obtained core/shell capsules, we further prepared hollow capsules by dissolving the core in hydrochloric acid: the powder of core/shell capsules was dispersed in distilled water, and hydrochloric acid was added until the $\mathrm{pH}$ value became 1.0, followed by stirring for up to $12 \mathrm{~h}$. After the separation of the hollow particles by filtration, they were dried under vacuum at room temperature for more than $72 \mathrm{~h}$.

\section{Microscopy and Calorimetry}

Morphology and sizes of the capsules were investigated by TEM on a microscope Jeol JEM-2000FXII operated with an acceleration voltage of $200 \mathrm{kV}$. The sample powder was dispersed in distilled water with sonication, and the sample was deposited onto a microgrid covered with a carbon-coated nitrocellulose membrane. No shadowing was applied.

Glass transition behaviors were investigated by using a DSC calorimeter Perkin Elmer Pyris Diamond. The temperature and heat flow were calibrated by using an indium standard, and the measurements were conducted under a nitrogen atmosphere. Typical sample size was 5 - $10 \mathrm{mg}$. Step-scan heating mode, which is a kind of 
temperature modulated DSC was used to yield both the non-reversing heat flow and reversing heat capacity. ${ }^{21}$ In general, the former reflects kinetic events such as crystallization and enthalpy relaxation, while the latter reflects basic heat capacity of the sample. The step-scans were done with a temperature step of $2 \mathrm{~K}$, the rate in heating segment of $5 \mathrm{~K} \mathrm{~min}^{-1}$, and the holding time of $1.5 \mathrm{~min}$. In this study, reversing heat capacity was exclusively used to evaluate $T_{\mathrm{g}}$ and the heat capacity jump at $T_{\mathrm{g}}\left(\Delta C_{p}\right)$. The method of evaluation for these parameters was the same as described previously. ${ }^{15,21}$ Iterative measurements were performed for different specimens to evaluate standard deviations for $T_{\mathrm{g}}$ and $\Delta C_{p}$.

\section{RESULTS}

Figures 1, 2, and 3 show typical electron micrographs of the obtained core/shell and hollow capsules. Rod-like core/shell structure is clearly seen in e.g., Figure 1 (b): dark parts indicate calcium carbonate cores. We found that the surface of BS-P whisker is well covered with the polymer shell and that few defects of the shell were observed. We found that the coverage becomes better as the degree of crosslink increases. However, for some capsules, end part of the core was lost as shown in Figure 2 (a). This was probably caused by the sonication which was applied before the TEM observation. The hollow particles were successfully obtained as shown in these micrographs. We made sure that no residual calcium carbonate was detected for the hollow samples by Fourier transform infrared spectroscopy and wide angle X-ray diffraction. Also, we made sure by scanning electron microscopy that the hollow capsules have not been collapsed after the removal of the core as described in the previous paper. $^{12}$

The removal of the core is achieved by the transportation of $\mathrm{CO}_{2}$ and $\mathrm{Ca}^{2+}$ through the polystyrene shell provided that there are no defects of the shell. We evaluated the translational diffusion coefficient of $\mathrm{Ca}^{2+}$ in polystyrene (without crosslink) to be $8.8 \times$ $10^{-19} \mathrm{~m}^{2} \mathrm{~s}^{-1}$ at $20^{\circ} \mathrm{C}^{22}$ This gives a diffusion time of $2.8 \times 10^{3} \mathrm{~s}$ for a distance of 50 $\mathrm{nm}$, which seems to be consistent with our experience that several hours were needed to remove the core completely. This result indicates that the surface of BS-P is well covered with the shell. We also found that longer time is needed to remove completely the core for samples with higher degrees of crosslink.

The thickness of polymer shell $d$ for the core/shell and hollow capsules was measured by TEM as shown in Table 2. Below, the core/shell and hollow samples are designated by '-CS' and '-H', respectively (see Table 2). We see that the thickness of the shell increases after the removal of the core. We found that this is associated with 
shrinkage of the capsule as a whole as we had shown qualitatively from measurement of the diameter of the capsule. ${ }^{12}$ However, quantitative analysis was not done because of the broad size distribution for the original BS-P particles. The thickening suggests certain stress existing in the shell, which can be released by structural relaxation during the removal of the core. The results in Table 2 show that this tendency of thickening is weakened as the degree of crosslink increases. The structural relaxation associated with the removal of the core may be hard to occur for highly crosslinked shells because of the structural stability induced by the crosslink.

The shell thickness of PSW5S-CS is greater than that of PSW5-CS by a factor of about 3. In Figure 3 (a), apparently thicker shells are observed. Thus the thickness of the shell can be controlled by the amount of monomers with respect to the whisker at least in the present range of thickness.

Figures 4 and 5 show typical traces for reversing heat capacity obtained by the step-scan DSC measurements. The top panels of these figures also include traces of non-reversing heat flow (dotted curves), which shows no apparent signal in the glass transition region. No endotherm due to enthalpy recovery (enthalpy relaxation) was observed that is usually seen for bulk polystyrene. This is the case for all samples of the present shell crosslinked capsules. In contrast, non-crosslinked hollow capsules, exothermic peaks are observed as reported in the previous study, ${ }^{12}$ which indicates that collapse of the hollow structure into a bulk-like material occurs. We found that the shell crosslinked hollow particles are stable enough to show a heat capacity trace of the second heating scan which is almost identical to that of the first heating of up to $145^{\circ} \mathrm{C}$.

Table 2 summarizes obtained $T_{\mathrm{g}}$ 's for the capsules. We see that $T_{\mathrm{g}}$ increases with increasing $x_{c}$ (degree of crosslink), and that the core/shell capsules generally show higher $T_{\mathrm{g}}$ 's than the corresponding hollow samples. Note that the latter trend is not observed for the non-crosslinked sample and the sample with $x_{\mathrm{c}}=0.018$ (PSW0 and PSW2). Table 2 also lists $T_{\mathrm{g}}$ 's of the bulk which were evaluated from independent experiments. $^{23}$ The $T_{\mathrm{g}}$ 's of the shell crosslinked capsules are generally higher than those of the bulk, while $T_{\mathrm{g}}$ 's identical to the bulk value are observed for PSW0. Comparing the results for PSW5 and PSW5S, we see that a shell with a greater $d$ exhibits a lower $T_{\mathrm{g}}$. Comparing the results for PSW5 and PSW5N samples, we see that the shells without coupling agent exhibit lower $T_{\mathrm{g}}$ 's. We should also note from the traces in Figures 4 and 5 that the temperature width of glass transition seems to increase with increasing $x_{\mathrm{c}}$.

$\Delta C_{p}$ for the hollow samples tends to decrease with increasing $x_{\mathrm{c}}$. This tendency was also reported for bulk crosslinked polystyrenes. ${ }^{23}$ As for the core/shell samples, $\Delta C_{p}$ 
values with sufficient accuracy were not obtained because of their small values, thus no data are shown in Table 2.

\section{DISCUSSION}

The above results revealed that $T_{\mathrm{g}}$ for the shell crosslinked capsules is varied by the degree of crosslink, the status of the core/shell interface, and the thickness of the shell. The increase in $T_{\mathrm{g}}$ with $x_{\mathrm{c}}$ reflects reduction of segmental mobility by the restriction due to crosslink. The core/shell capsule with the highest $x_{\mathrm{C}}$ (PSW10-CS) did not exhibit any $T_{\mathrm{g}}$ signal up to $220^{\circ} \mathrm{C}$ : the reason for this is not clear, but the $T_{\mathrm{g}}$ might be too high to be detected. On the other hand, the corresponding hollow sample (PSW10-H) shows a $T_{\mathrm{g}}$ at about $124^{\circ} \mathrm{C}$.

Below, we further discuss the molecular interaction at the interface, heterogeneity of crosslink, and configuration of network polymer in ultrathin layer to interpret the current results.

\section{Interfacial Effect}

The difference in $T_{\mathrm{g}}$ between the core/shell and hollow capsules may indicate that the interaction at the interface between the polymer and calcium carbonate affects $T_{\mathrm{g}}$ significantly. This is further supported that little difference in $T_{\mathrm{g}}$ is seen between the core/shell and hollow capsules for PSW5N (capsule without the coupling agent). We found no significant difference in morphology at the interface from the TEM images: we observed no defects such as gap between the core and shell for PSW5N-CS. The difference of the interface induced by MAMSP may be relevant to structure on a molecular level. MAMSP is expected to couple with hydroxyl groups on the surface of whisker forming (polymer)-Si-O-(whisker) linkages. ${ }^{12}$ It is thus suggested that good compatibility at the interface restricts the segmental relaxation in the interfacial region, which leads to higher $T_{\mathrm{g}}$ 's for the core/shell capsules.

The difference in $T_{\mathrm{g}}$ between the core/shell and hollow capsules was very little for PSW0 and PSW2 in spite of the presence of MAMSP. This indicates that the effect of interface is enhanced by crosslink. Fryer et al. studied the effect of interaction between the polymer and substrate on the $T_{\mathrm{g}}$ of supported ultrathin films, and concluded that high interfacial energy leads to higher $T_{\mathrm{g}}$ than the bulk $T_{\mathrm{g}}{ }^{19}$ If the difference in $T_{\mathrm{g}}$ between the core/shell and hollow capsules is dominated by the interfacial energy, the present result indicates that the interfacial energy increases with increasing $x_{\mathrm{c}}$. Unfortunately, we can not check this point further at the present time, because we have no available data for the interfacial energy parameters of crosslinked polystyrenes. 
This problem may be relevant to the heterogeneous distribution of crosslink points which will be discussed in the following section: the crosslink density in the interfacial region might be different from the inner part of the shell, and such heterogeneity might depend on $x_{\mathrm{c}}$.

The effect of interface is also reflected in the result that thicker shells (PSW5S) exhibit only a slight difference in $T_{\mathrm{g}}$ between the core/shell and hollow capsules. The interfacial effects are expected to be reduced as the thickness increases. We should note here, that the error (standard deviation) for $d$ listed in Table 2 indicates rather broad size distribution. For further discussion on the thickness dependence of $T_{\mathrm{g}}$, experiments on samples with narrower size distribution are to be done. Refinement of the sample preparation method will be done in our future work.

The $T_{\mathrm{g}}$ variation with the thickness of polymer ultrathin films has been extensively studied. $^{7}$ In the case of free-standing thin films, the $T_{\mathrm{g}}$ decreases with decreasing thickness, ${ }^{2,4}$ while for supported films the $T_{\mathrm{g}}$ increases or decreases depending upon the materials of both the polymer and substrate. ${ }^{16}$ The present thin shells exhibit higher $T_{\mathrm{g}}$ 's than the bulk values. In this case, higher interfacial energy between the crosslinked polystyrene and calcium carbonate is expected. ${ }^{19}$ Hence, the $T_{\mathrm{g}}$ is expected to increase as the thickness of shell decreases. This is evidenced by the fact that PSW5-CS shows a higher $T_{\mathrm{g}}$ than that for PSW5S-CS.

The interfacial interaction between polystyrene and calcium carbonate is considered to be originated from weak non-bonded interactions. On the other hand, MAMSP is expected to enhance the interfacial interaction as mentioned above. The present results on $T_{\mathrm{g}}$ show stronger interfacial interactions for the crosslinked capsules than the non-crosslinked capsule. Thus, the crosslink might enhance the effect of MAMSP, although the mechanism is not known at present.

\section{Effects of Configuration and Heterogeneous Crosslink}

As we discussed in the previous section, the interfacial effect on $T_{\mathrm{g}}$ may be significant. However, the lower $T_{\mathrm{g}}$ 's for the hollow capsules than for the core/shell capsules should not be attributed only to the interfacial effect. For example, structural relaxation is suggested to occur by the thickening which is observed after the removal of the core. The configuration of crosslinked shell for hollow capsule may be different from that of core/shell capsule. Therefore, we should consider some additional effects other than the interfacial interaction: these may be specific to crosslinked ultrathin layer. Here, we consider two effects, i.e., configuration of the polymer in thin layer and heterogeneous crosslink. These are suggested by the following findings: (1) hollow 
capsule is a counterpart of free-standing thin film, therefore, reduction of $T_{\mathrm{g}}$ from the bulk value would be observed, but actually, increase in $T_{\mathrm{g}}$ was observed. (2) PSW5-H exhibits higher $T_{\mathrm{g}}$ than PSW5S-CS and -H despite that these three have nearly the same thickness. (3) No difference in $T_{\mathrm{g}}$ from the bulk value is observed for PSW0. Broadness of the glass transition increases with increasing $x_{c}$. Unfortunately, to our knowledge, no experimental data have been reported for the glass transition of crosslinked polymer ultrathin (flat) films, which would be a support for our speculation. It might be possible that the geometry of nano-sized polymer material affects the configuration, which results in different segmental dynamics. This is suggested for example, by the fact that the non-crosslinked hollow capsule (PSW0-H) shows nearly the same $T_{\mathrm{g}}$ as the bulk value, while a free-standing film with the same thickness (33 $\mathrm{nm}$ ) exhibits a $T_{\mathrm{g}}$ reduction of ca. $20 \mathrm{~K}^{4}{ }^{4}$ To elucidate the origin of this difference, measurements on capsules with various shapes and sizes would be required.

The broadness of $T_{\mathrm{g}}$ for highly crosslinked shells shown in Figures 4 and 5 may be due to the intrinsic feature of the network itself and/or the heterogeneous crosslink. The former effect is relevant to the characteristic length of dynamic glass transition, ${ }^{24,25}$ which has been revealed to decrease with increasing $x_{\mathrm{c}}$ for bulk materials. ${ }^{23}$ The number of available configurations deceases significantly with increasing $x_{\mathrm{c}}$ for network polymer, and this effect is enhanced by crosslink which is responsible for the $T_{\mathrm{g}}$ variation with respect to $x_{\mathrm{c}}$.

The effect of configuration on $T_{\mathrm{g}}$ has been discussed to understand the different segmental dynamics in nano-sized glass-formers including freeze-dried polymers. ${ }^{3,5,7,15,26-30}$ The idea of configuration effects seems to be important to interpret the fact that only a single glass transition signal is observed at a temperature different from the bulk $T_{\mathrm{g}}$ for ultrathin films (e.g., free-standing polystyrene films). The idea of the surface layer with a higher mobility than the bulk (surface effect) $)^{4,15,17,31-33}$ may be another important cause for the $T_{\mathrm{g}}$ depression in free-standing ultrathin films. ${ }^{4,7}$ This assumes a layered structure from which a multi-step transition may be expected. However, in practice, multiple transition was observed only for

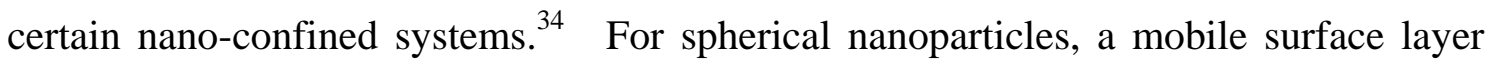
has been suggested to exist by reduced $\Delta C_{p}$ values, but no distinct $T_{\mathrm{g}}$ signal of the mobile layer has been observed. ${ }^{15}$ It is an open question as to how the distinct regions with different segmental dynamics couple with each other so that a single $T_{\mathrm{g}}$ signal is exhibited as in the case of ultrathin films.

The present thin shells exhibit increased $T_{\mathrm{g}}$ 's rather than decreased $T_{\mathrm{g}}$ 's. This suggests that the surface effect on $T_{\mathrm{g}}$ is not very significant for the present capsule 
system. The surface effect of depressing $T_{\mathrm{g}}$ might be defeated by the other effects of such as the interfacial interaction, configuration, and heterogeneous crosslink.

In the present thin shells, the configuration might not be far from equilibrium as is suggested from the TEM images: the outer surface of the capsules seems to be rather rough as shown in Figure 1 (b). Probably, such rough surface has been formed during the polymerization so as to stabilize the configuration to some extent. However, for highly crosslinked shells, such stabilization may be restricted, which may be a cause of $T_{\mathrm{g}}$ increase from the bulk values as observed in this study.

Spatially heterogeneous distribution of the crosslink segments is suggested from the broadness of the glass transition as we mentioned above. In general, such heterogeneity of crosslink was suggested for bulk materials. ${ }^{35-37}$ This gives rise to some region which has a higher density of crosslink than $x_{c}$. This might be another cause of the observed increase in $T_{\mathrm{g}}$ for the crosslinked shells from the bulk value. A simulation study showed that the local density is increased in the vicinity of interface for a confined polymer system. ${ }^{38}$ Such heterogeneous distribution induced by an interface might lead to a localization of crosslink points in certain regions. It is likely that the heterogeneity of crosslink in thin layer is greater than that in the bulk state, and that highly crosslinked regions in the shell are responsible for the higher $T_{\mathrm{g}}$ than the bulk value as well as for the broadened $T_{\mathrm{g}}$.

\section{SUMMARY AND CONCLUSION}

We have successfully prepared shell crosslinked capsules with different crosslink densities and thicknesses of less than $100 \mathrm{~nm}$ using calcium carbonate whisker as a core material. The obtained calorimetric data show that (1) core/shell capsules exhibit higher $T_{\mathrm{g}}$ 's than the hollow capsules, (2) crosslinked thin shells exhibit higher $T_{\mathrm{g}}$ 's than the bulk values, (3) $T_{\mathrm{g}}$ tends to decrease with increasing $d$ for core/shell capsules with $x_{\mathrm{C}}$ $=0.048$, (4) the coupling agent enhances $T_{\mathrm{g}}$ for core/shell capsules, and (5) the broadness of $T_{\mathrm{g}}$ increases with increasing $x_{\mathrm{c}}$. It is revealed that the trends of (1), (2), and (3) become salient for highly crosslinked shells. These results suggest that the molecular interaction at the interface that restricts segmental relaxation, configurational restriction of the network chains (confinement in a thin layer), and heterogeneity of crosslink affect significantly the $T_{\mathrm{g}}$ of the present thin shells. The molecular mechanism of the observed effects of crosslink is not yet elucidated. In addition, it is not known how the shape of the capsules affects the segmental dynamics in the shell. Further investigations should be done to elucidate these points by preparing capsules with various geometries and with narrower size distributions. 
ACKNOWLEDGMENT. The authors thank Maruo Calcium Co., Japan, for supplying the calcium carbonate whisker.

\section{REFERENCES AND NOTES}

1. Keddie, J. L.; Jones, R. A. L.; Cory, R.A. Europhys Lett 1994, 27, 59; Faraday

Discuss 1994, 98, 219.

2. Forrest, J. A.; Dalnoki-Veress, K.; Dutcher, J. R. Phys Rev E 1997, 56, 5705.

3. Ngai, K. L.; Rizos, A. K.; Plazek, D. J. J Non-Cryst Solids 1998, 235-237, 435.

4. Mattsson, J.; Forrest, J. A.; Börjesson, L. Phys Rev E 2000, 62, 5187.

5. de Gennes, P. G. Eur Phys J. E 2000, 2, 201.

6. Long, D.; Lequeux, F. Eur Phys J E 2001, 4, 371.

7. Forrest, J. A.; Dalnoki-Veress, K. Adv Colloid Interface Sci 2001, 94, 167.

8. Thurmond II, K. B.; Kowalewski, T.; Wooley, K. L. J Am Chem Soc 1997, 119, 6656.

9. Radtchenko, I. L.; Sukhorukov, G. B.; Leporatti, S.; Khomutov, G. B.; Donath, E.;

Möhwald, H. J Colloid Interface Sci 2000, 230, 272.

10. Jönsson, J.-E.; Karlsson, O. J.; Hassander, H.; Törnell, B. Macromolecules 2001, 34, 1512.

11. Harada, A.; Kataoka, K. Science 1999, 283, 65.

12. Sasaki, T.; Kitagawa, T.; Sato, S.; Irie, S.; Sakurai, K. Polym J 2005, 37, 434.

13. Gauer, U.; Wunderlich, B. Macromolecules 1980, 13, 1618.

14. Ming, W.; Zhao, J.; Lu, X.; Wang, C.; Fu, S. Macromolecules 1996, 29, 7678.

15. Sasaki, T.; Shimizu, A.; Mourey, T. H.; Thurau, C. T.; Ediger, M. D. J Chem Phys 2003, 119, 8730.

16. Fryer, D. S.; Nealey, P. F.; de Pablo, J. J. Macromolecules 2000, 33, 6439.

17. Kim, J. H.; Jang, J.; Zin, W.-C. Langmuir 2000, 16, 4064.

18. Torres, J. A.; Nealey, P. F.; de Pablo, J. J. Phys Rev Lett 2000, 85, 3221.

19. Fryer, D. S.; Peters, R. D.; Kim, E. J.; Tomaszewski, J. E.; de Pablo, J. J.; Nealey, P. F.; White, C. C.; Wu, W. Macromolecules 2001, 34, 5627.

20. Kobayashi, K.; Kasahara, H. Jpn Patent 2001, 98164. We did not use the other coupling agent, 2-hydroxymethyl methacrylate in this study, because it was less effective than MAMSP in improving the interfacial compatibility.

21. Sasaki, T.; Yamauchi, N.; Irie, S.; Sakurai, K. J Polym Sci B: Polym Phys 2005, 43, 115.

22. To evaluate the diffusion coefficient, aqueous solution of $\mathrm{Ca}^{2+}$ and distilled water was separated by a $1.5 \mu \mathrm{m}$ thick polystyrene film prepared by spin-coating, and the 
time evolution of $\left[\mathrm{Ca}^{2+}\right]$ was recorded over 30 days.

23. Sasaki, T.; Uchida, T.; Sakurai, K. J Polym Sci B: Polym Phys 2006, 44, in press.

24. Ediger, M. D.; Angell, C. A.; Nagel, S. R. J Phys Chem 1996, 100, 13200.

25. Hemmpel, E.; Hensel, A.; Schick, C.; Donth, E. J Phys Chem B 2000, 104, 2460.

26. Ding, J.; Xue, G.; Dai, Q.; Cheng, R. Polymer 1993, 34, 3325.

27. Bernazzani, P.; Simon, S. L.; Plazek, D.J.; Ngai, K. L. Eur Phys J E 2002, 8, 201.

28. Grohens, Y.; Hamon, L.; Reiter, G.; Soldera, A.; Holl, Y. Eur Phys J E 2002, 8, 217.

29. Ngai, K. L. Eur Phys J E 2002, 8, 225.

30. Simon, S. L.; Bernazzani, P.; McKenna, G. B. Polymer 2003, 44, 8025.

31. Fukao, K.; Miyamoto, Y. Phys Rev E 2000, 61, 1743.

32. Sharp, J. S.; Forrest, J. A. Phys Rev Lett 2003, 91, 235701.

33. Akabori, K.; Tanaka, K.; Nagamura, T.; Takahara, A.; Kajiyama, T. Macromolecules 2005, 38, 9735.

34. Park, J.-Y.; McKenna, G. B. Phys Rev B 2000, 61, 6667.

35. Kannurpatti, A. R.; Anderson, K. J.; Anseth, J. W.; Bowman, C. N. J Polym Sci B: Polym Phys 1997, 35, 2297.

36. Scott, T. F.; Cook, W. D.; Forsythe, J. S. Eur Polym J 2002, 38, 705.

37. Alves, N. M.; Gomez Ribelles, J. L.; Mano, J. F. Polymer 2005, 46, 491.

38. Baschnagel, J.; Binder, K. Macromolecules 1995, 28, 6808.

\section{FIGURE CAPTIONS}

Figure 1. Transmission electron micrographs for (a) PSW2-CS, (b) PSW2-CS observed with a higher magnification, and (c) PSW2-H. The outer polymer shell is clearly seen in (b).

Figure 2. Transmission electron micrographs for (a) PSW10-CS and (b) PSW10-H.

Figure 3. Transmission electron micrographs for (a) PSW5S-CS and (b) PSW5S-H.

Figure 4. Typical DSC traces of reversing heat capacity for the core/shell capsules obtained by the step-scan measurements. The thin lines indicate extrapolations from the glassy and liquid states to the glass transition region. The dotted curve in (a) indicates non-reversing heat flow trace.

Figure 5. Typical DSC traces of reversing heat capacity for the hollow capsules. The dotted curve in (a) indicates non-reversing heat flow trace. 
Table 1. Mass ratios of the agents fed to prepare core/shell capsules ${ }^{a}$

\begin{tabular}{llll}
\hline sample & $x_{\mathrm{C}}{ }^{\mathrm{b}}$ & $m_{\mathrm{S}} / m_{\mathrm{BS}-\mathrm{P}}$ & $m_{\text {MAMSP }} / m_{\mathrm{S}}$ \\
\hline PSW0 & 0 & 0.5 & 0.005 \\
PSW2 & 0.018 & 0.5 & 0.005 \\
PSW5 & 0.048 & 0.5 & 0.005 \\
PSW5S & 0.048 & 1.5 & 0.005 \\
PSW5N & 0.048 & 0.5 & 0 \\
PSW7 & 0.066 & 0.5 & 0.005 \\
PSW10 & 0.092 & 0.5 & 0.005 \\
\hline
\end{tabular}

${ }^{a} m_{\mathrm{s}}, m_{\mathrm{BS}-\mathrm{P}}$, and $m_{\mathrm{MAMSP}}$ are the masses of styrene, BS-P (whisker), and MAMSP, respectively.

${ }^{\mathrm{b}}$ degree of crosslink specified by $x_{\mathrm{C}}=m_{\mathrm{DVB}} /\left(m_{\mathrm{S}}+m_{\mathrm{DVB}}\right)$ 
Table 2. Thickness, glass transition temperature, and heat capacity change at $T_{\mathrm{g}}$ for the outer shell

\begin{tabular}{llllll}
\hline sample & & $d(\mathrm{~nm})$ & $T_{\mathrm{g}}\left({ }^{\circ} \mathrm{C}\right)$ & $\Delta C_{p}\left(\mathrm{~J} \mathrm{~g}^{-1} \mathrm{~K}^{-1}\right)$ & $T_{\mathrm{g}}{ }^{\text {bulk }}\left({ }^{\circ} \mathrm{C}\right)^{\mathrm{a}}$ \\
\hline \multirow{2}{*}{ PSW0 $^{\mathrm{b}}$} & $-\mathrm{CS}^{\mathrm{c}}$ & $29 \pm 11$ & $101 \pm 2.0$ & & 98.2 \\
& $-\mathrm{H}^{\mathrm{d}}$ & $33 \pm 9$ & $99 \pm 2.0$ & 0.28 & 98.2 \\
PSW2 & $-\mathrm{CS}$ & $50 \pm 15$ & $105.8 \pm 2.2$ & & 101.3 \\
& $-\mathrm{H}$ & $73 \pm 21$ & $105.5 \pm 1.0$ & $0.25 \pm 0.06$ & 101.3 \\
PSW5 & $-\mathrm{CS}$ & $26 \pm 8$ & $126.4 \pm 0.8$ & & 106.2 \\
& $-\mathrm{H}$ & $76 \pm 44$ & $120.1 \pm 1.9$ & $0.21 \pm 0.06$ & 106.2 \\
PSW5S & $-\mathrm{CS}$ & $81 \pm 15$ & $112.9 \pm 0.5$ & & 106.2 \\
& $-\mathrm{H}$ & $79 \pm 6$ & $111.6 \pm 1.1$ & $0.20 \pm 0.03$ & 106.2 \\
PSW5N & $-\mathrm{CS}$ & $42 \pm 23$ & $114.4 \pm 2.1$ & & 106.2 \\
& $-\mathrm{H}$ & $57 \pm 26$ & $111.8 \pm 1.0$ & $0.21 \pm 0.03$ & 106.2 \\
PSW7 & $-\mathrm{CS}$ & $26 \pm 6$ & $130.2 \pm 2.6$ & & 109.2 \\
& $-\mathrm{H}$ & $44 \pm 17$ & $125.7 \pm 1.0$ & $0.26 \pm 0.02$ & 109.2 \\
PSW10 & $-\mathrm{CS}$ & $44 \pm 18$ & $\mathrm{NA}$ & & 113.5 \\
& $-\mathrm{H}$ & $57 \pm 21$ & $124.0 \pm 0.9$ & $0.15 \pm 0.06$ & 113.5 \\
\hline
\end{tabular}

\footnotetext{
${ }^{\mathrm{a}} T_{\mathrm{g}}$ in the bulk state evaluated from the data in Ref 23

b data from Ref 12

c core/shell capsule

${ }^{\mathrm{d}}$ hollow capsule

e no $T_{\mathrm{g}}$ signal up to $220^{\circ} \mathrm{C}$
} 
(a)

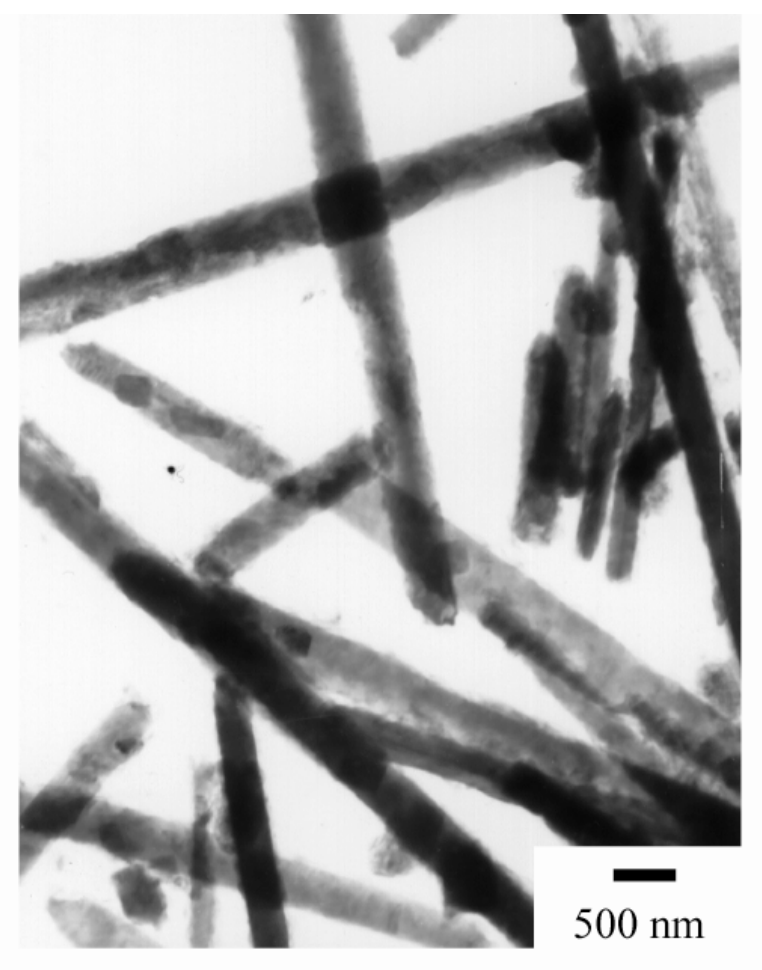

(b)

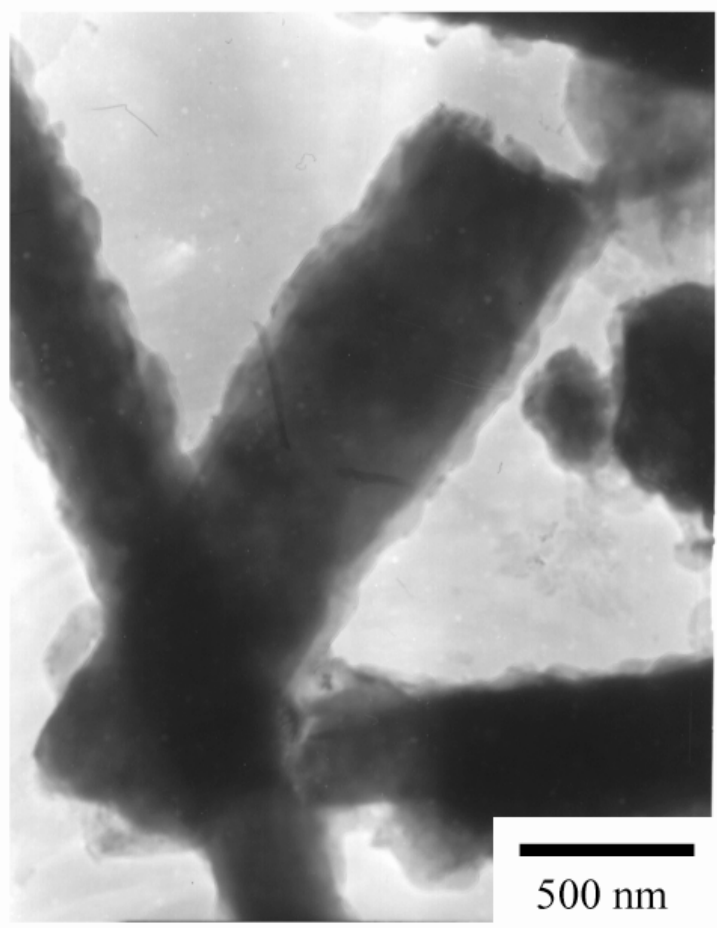

Figure 1 (a), (b). T. Sasaki et al. 
(c)

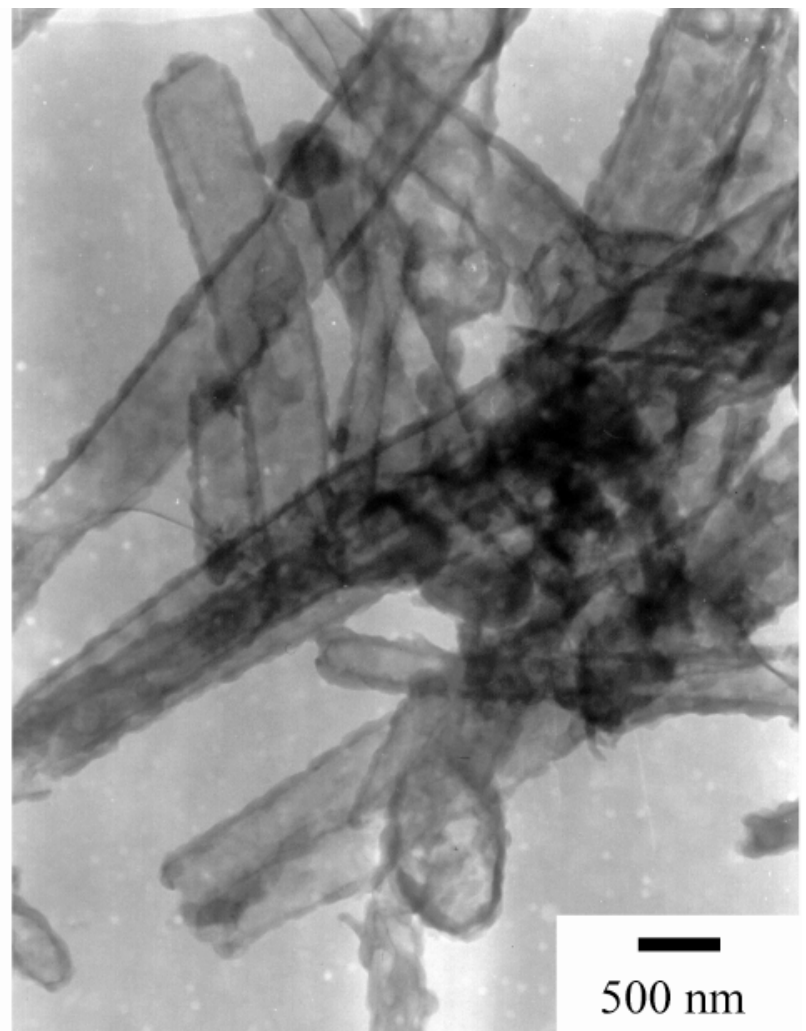

Figure 1 (c). T. Sasaki et al. 
(a)

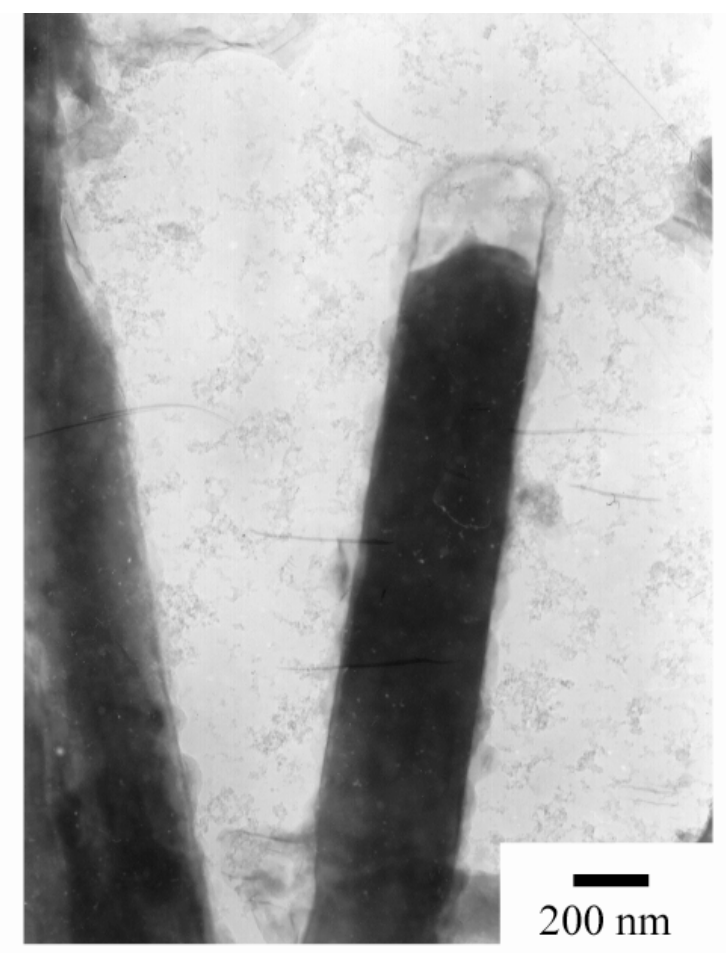

(b)

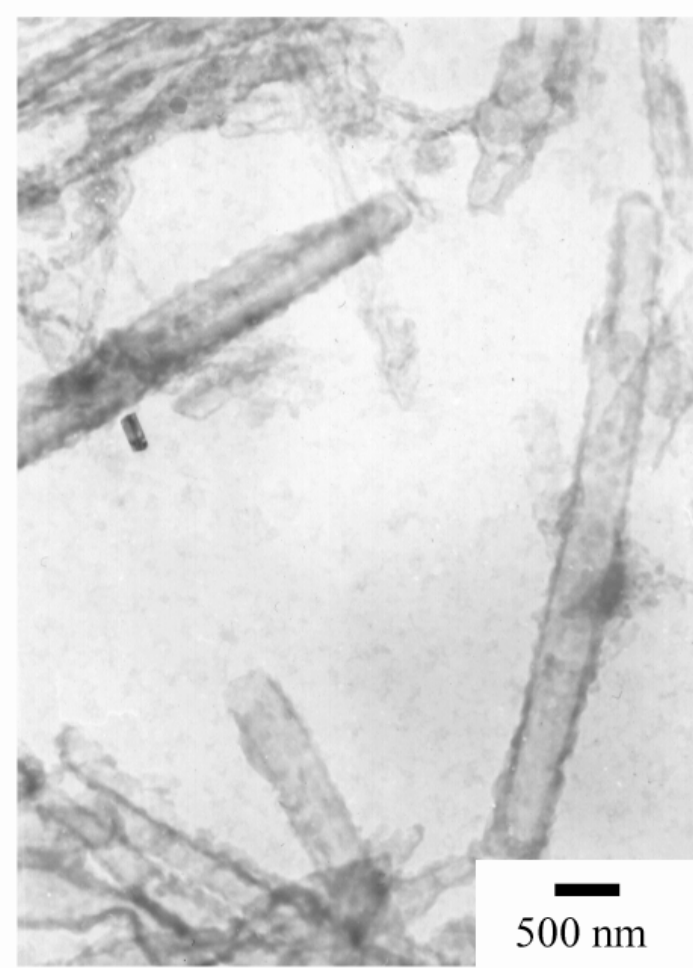

Figure 2. T. Sasaki et al. 
(a)

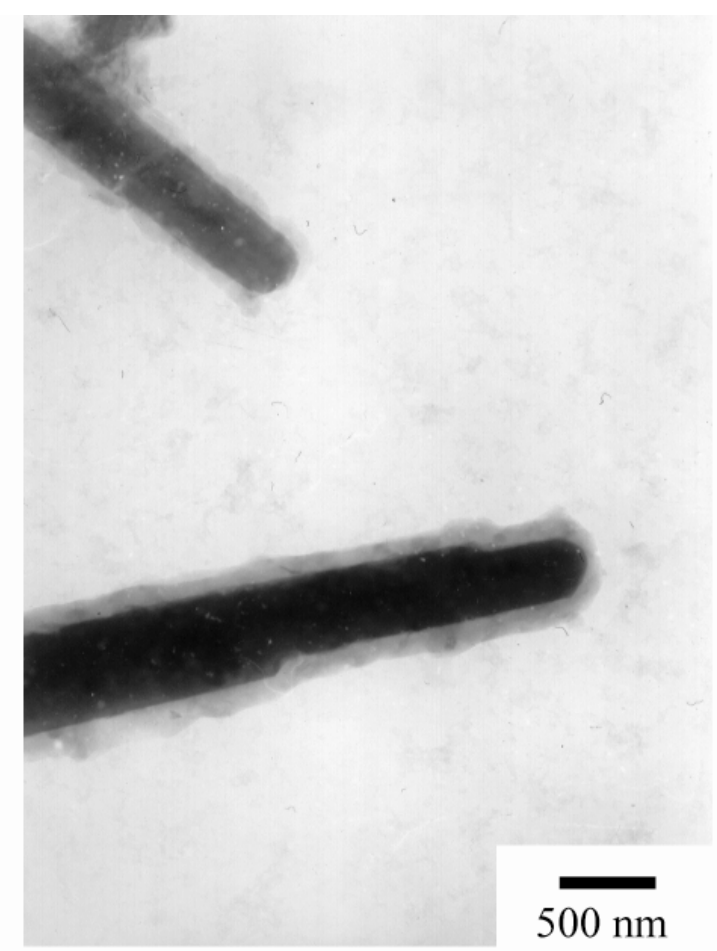

(b)

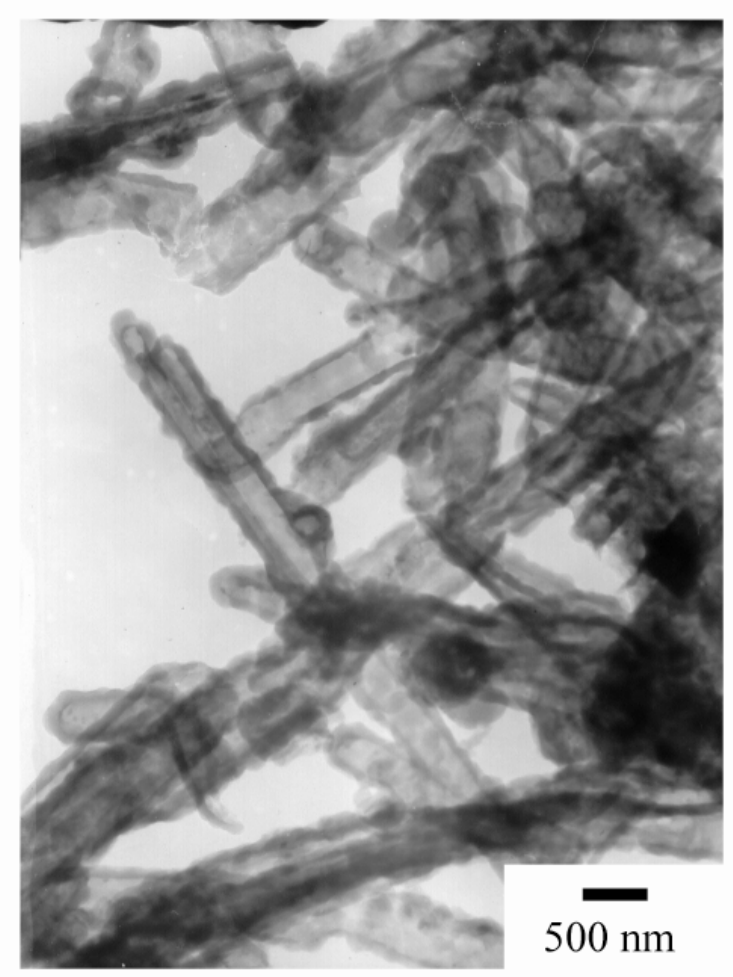

Figure 3. T. Sasaki et al. 


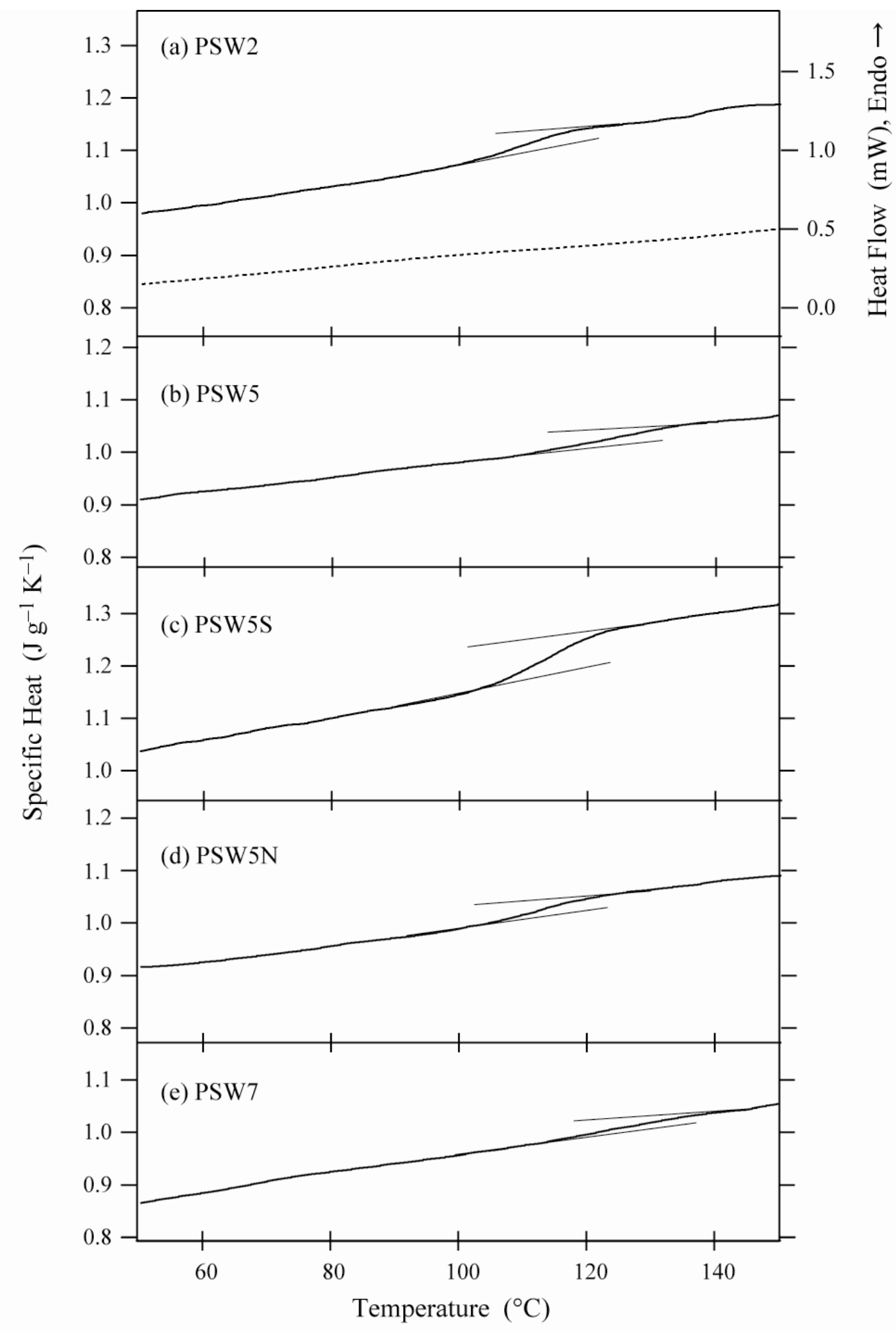

Figure 4. T. Sasaki et al. 


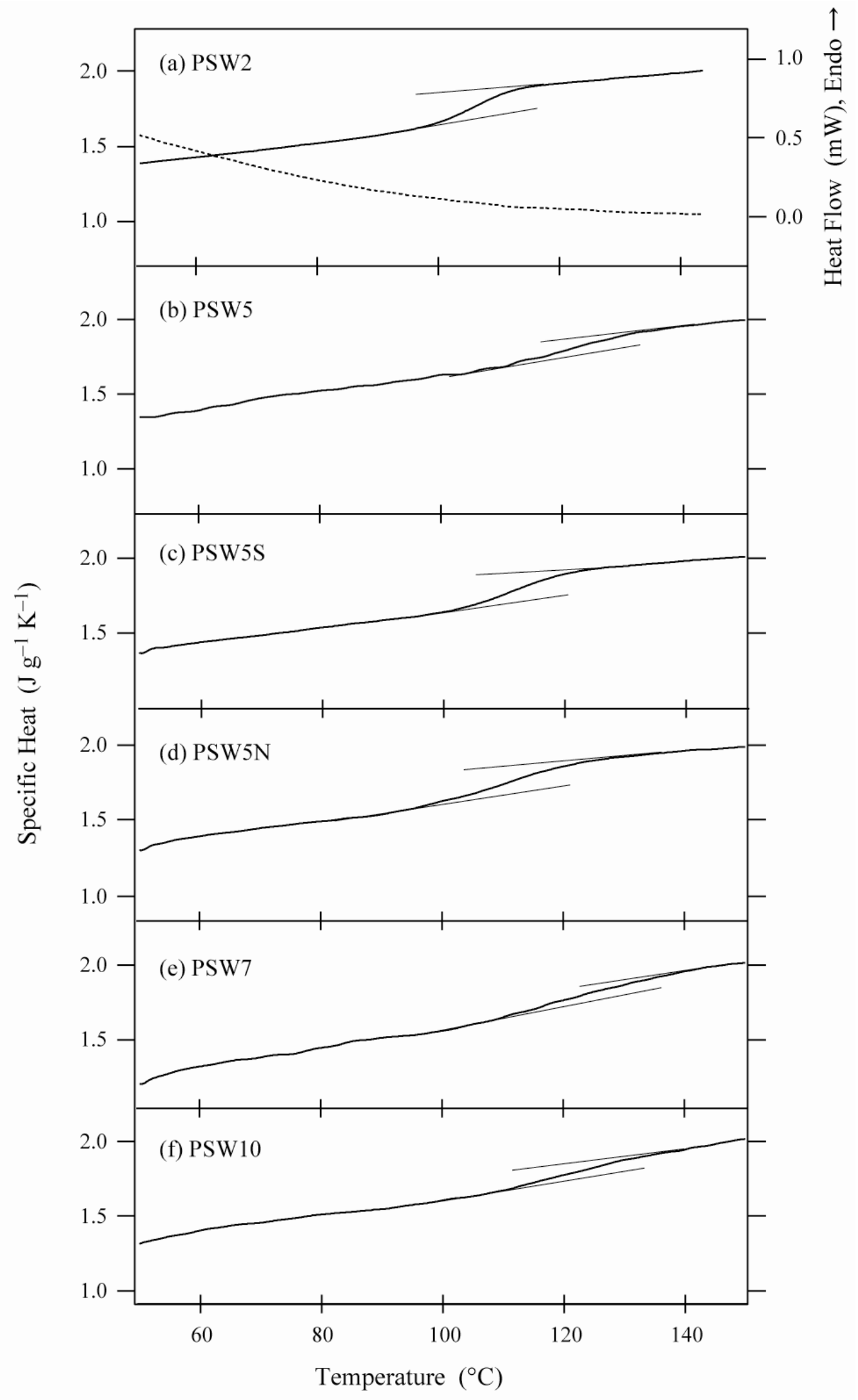

Figure 5. T. Sasaki et al. 
